\title{
Type 2 diabetes and atrophy of medial temporal lobe structures on brain MRI
}

\author{
T. den Heijer ${ }^{1,2}$, S. E. Vermeer ${ }^{1,2}$, E. J. van Dijk ${ }^{1,2}$, N. D. Prins ${ }^{1,2}$, P. J. Koudstaal ${ }^{1,2}$, A. Hofman ${ }^{1}$, \\ M. M. B. Breteler ${ }^{1}$ \\ ${ }^{1}$ Department of Epidemiology and Biostatistics, Erasmus Medical Center, Rotterdam, The Netherlands \\ ${ }^{2}$ Department of Neurology, Erasmus Medical Center, Rotterdam, The Netherlands
}

\section{Abstract}

Aim/hypothesis. Type 2 diabetes increases the risk not only of vascular dementia but also of Alzheimer's disease. The question remains whether diabetes increases the risk of Alzheimer's disease by diabetic vasculopathy or whether diabetes influences directly the development of Alzheimer neuropathology. In vivo, hippocampal and amygdalar atrophy on brain MRI are good, early markers of the degree of Alzheimer neuropathology. We investigated the association between diabetes mellitus, insulin resistance and the degree of hippocampal and amygdalar atrophy on magnetic resonance imaging (MRI) accounting for vascular pathology.

Methods. Data was obtained in a population-based study of elderly subjects without dementia between 60 to 90 years of age. The presence of diabetes mellitus and, in non-diabetic subjects, insulin resistance was assessed for 506 participants in whom hippocampal and amygdalar volumes on MRI were measured. We assessed the degree of vascular morbidity by rating carotid atherosclerosis, and brain white matter lesions and infarcts on MRI.

Results. Subjects with diabetes mellitus had more hippocampal and amygdalar atrophy on MRI compared to subjects without diabetes mellitus. Furthermore, increasing insulin resistance was associated with more amygdalar atrophy on MRI. The associations were not due to vascular morbidity being more pronounced in persons with diabetes mellitus.

Conclusions/interpretation. Type 2 diabetes is associated with hippocampal and amygdalar atrophy, regardless of vascular pathology. This could suggest that Type 2 diabetes directly influences the development of Alzheimer neuropathology. [Diabetologia (2003) 46:1604-1610]

Keywords Type 2 diabetes, brain, dementia, epidemiology, hippocampus, amygdala, memory, MRI, insulin, glucose.
Type 2 diabetes increases the risk of stroke [1] and vascular dementia [2]. Recently, patients with Type 2 diabetes were found to have an increased risk of the most common form of dementia, Alzheimer's disease

Received: 11 June 2003 / Revised: 8 August 2003

Published online: 1 November 2003

(C) Springer-Verlag 2003

Corresponding author: Dr. M. M. B. Breteler, Department of Epidemiology and Biostatistics, Erasmus Medical Center, Dr Molewaterplein 50, PO Box 1738, 3000 DR Rotterdam, The Netherlands

E-mail: m.breteler@erasmusmc.nl

Abbreviations: MRI, magnetic resonance imaging; APOE, Apolipoprotein E.
$[3,4]$. The pathophysiological mechanism of the relation between diabetes mellitus and Alzheimer's disease is not clear. Diabetic vasculopathy can cause cerebrovascular brain damage, which is frequently found in patients with Alzheimer's disease [5]. However, other, more direct effects of diabetes on the development of Alzheimer neuropathology can also be involved. Advanced glycation end products increase aggregation of proteins involved in Alzheimer's disease [6]. Furthermore, dysfunction of insulin signalling in the brain has been implicated in the pathogenesis of Alzheimer's disease [7]. The neuropathology of Alzheimer's disease occurs with greatest severity and in an early stage of the disease in the hippocampus and amygdala, brain structures in the medial temporal 
lobe [8]. In vivo assessment of hippocampal volume on magnetic resonance imaging (MRI) of the brain provides a good estimate of the degree of Alzheimer neuropathology, even in elderly subjects without clinical symptoms of dementia [9]. Several studies show that patients with mild Alzheimer's disease have smaller volumes of the hippocampus $[10,11,12]$ and amygdala $[13,14]$ on MRI compared to healthy control subjects. We examined the association between diabetes mellitus, insulin resistance and hippocampal and amygdalar atrophy on MRI using these as early MRI markers of Alzheimer's disease. We accounted for atherosclerosis and cerebrovascular disease to examine whether any association was caused by concomitant vascular disease.

\section{Methods}

Participants. The Rotterdam Study is a large population-based cohort study in the Netherlands that investigates the prevalence, incidence and determinants of chronic diseases in the elderly [15]. Baseline examinations were done in 1990 to 1993. In 1995 to 1996, we randomly selected 965 living members (60-90 years of age) of the cohort in strata of sex and age (5 years) to participate in the Rotterdam Scan Study, designed to investigate age-related brain abnormalities on MRI [16]. After excluding individuals who were demented $(n=17)$ [17] or had MRI contraindications $(n=116), 832$ people were eligible and invited. Among these, 563 participants gave their written informed consent and underwent MRI scanning of the brain (participation rate: $68 \%$ ). Participants were in general healthier than non-participants [18]. The study protocol was approved by the medical ethics committee of the Erasmus Medical Center, Rotterdam, The Netherlands.

Assessment of diabetes mellitus and insulin resistance. Presence of diabetes mellitus was assessed at the baseline of the Rotterdam Study (1990-1993) and at time of MRI (1995-1996). Participants where considered to have diabetes mellitus if they reported use of oral anti-diabetic treatment or of insulin, or if they had a random serum glucose concentration greater than or equal to $11.1 \mathrm{mmol} / \mathrm{l}$. In addition, if a postload glucose concentration ( $2 \mathrm{~h}$ after a glucose drink of $75 \mathrm{~g}$ in $200 \mathrm{ml}$ water) at baseline was greater than or equal to $11.1 \mathrm{mmol} / \mathrm{l}$ the participant was also considered to have diabetes mellitus. Glucose concentrations were measured by the glucose hexokinase method. Insulin resistance in non-diabetic subjects at baseline was assessed by the ratio of the post-load insulin concentration (Medgenix, Brussels, Belgium) over post-load glucose concentration.

MRI procedures. In 1995 to 1996, standard T1, T2 and protondensity weighted MR sequences of the brain were made using a 1.5 Tesla MR unit (VISION MR, Siemens, Erlangen, Germany) [19]. After these sequences were finished, an additional custom-made three-dimensional MRI sequence of the whole brain was acquired (named half-Fourier acquisition single-shot turbo spin echo [20]). This three-dimensional MRI sequence was used for later volumetric assessments of the hippocampus and amygdala. A total of 52 participants developed claustrophobia during the MRI scanning period, leaving 511 participants with a completed three-dimensional MRI sequence.

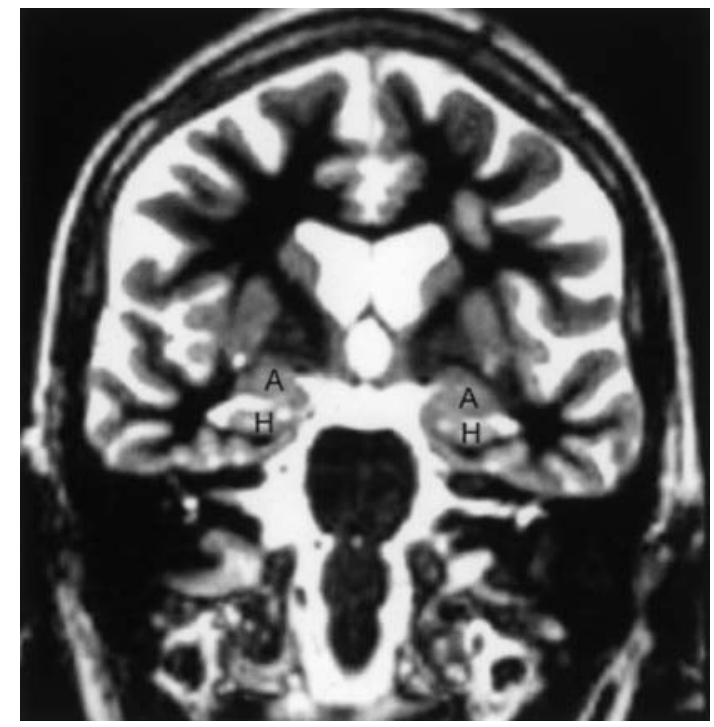

Fig. 1. Coronal MRI slice on which the hippocampus $(\mathrm{H})$ and amygdala (A) are depicted

Hippocampal and amygdalar volumes on MRI. For the 511 participants with a three-dimensional MRI sequence, we reformatted a series of coronal brain slices (contiguous $1.5-\mathrm{mm}$ slices) aligned to be perpendicular to the long axis of the hippocampus and the middle sagittal slice. The procedure of segmenting the hippocampus and amygdala has been described in detail [20]. Briefly, we manually traced the boundaries of the hippocampi and amygdalae using a mouse-driven pointer (Fig. 1), which yielded outlined areas $\left(\mathrm{mm}^{2}\right)$. We proceeded from posterior to anterior, starting on the slice where the crux of the fornices of the hippocampus was in full profile. We multiplied the summed areas with slice thickness $(1.5-\mathrm{mm})$ to calculate estimates of the left and right hippocampal and amygdalar volume $(\mathrm{ml})$. Total hippocampal and amygdalar volumes were calculated by summing the left and right hippocampal volume and the left and right amygdalar volume. As a proxy for head size, we measured on the middle sagittal MRI slice the intracranial cross-sectional area [20, 21]. We corrected for head size difference across the subjects as follows [13, 21]. First, each subject's hippocampal or amygdalar volumes were divided by their measured head size area. Next, to obtain head size corrected, normalised volumes the ratios for each subject were multiplied by the average head size area (men and women separately).

Other measurements. We interviewed and gave physical examinations to the participants to obtain information on their: educational level (according to UNESCO [22]), BMI (weight divided by the square of the height), pack-years of cigarette smoking, blood pressure and serum total cholesterol [23]. Memory function was evaluated with a 15 -word learning test which consisted of three immediate learning trials and a delayed recall trial [19]. For each participant, we calculated zscores (individual test score minus mean test score divided by the standard deviation). We constructed a compound score for memory performance by averaging the z-score of the three immediate recall trials and the delayed recall trial [19]. Apolipoprotein $\mathrm{E}(A P O E)$ genotyping yielded the following alleles: $\varepsilon 2$, $\varepsilon 3$, and $\varepsilon 4$ [24]. We classified participants into those with and without a $\varepsilon 4$ allele because the $\varepsilon 4$ allele is a strong risk factor of Alzheimer's disease [25]. Because the presence of the $\varepsilon 2$ allele can reduce the risk of Alzheimer's disease [25], we ex- 
Table 1. Characteristics of participants with and without diabetes mellitus

\begin{tabular}{|c|c|c|c|}
\hline Variable & $\begin{array}{l}\text { No diabetes mellitus } \\
n=465\end{array}$ & $\begin{array}{l}\text { Diabetes mellitus } \\
n=41\end{array}$ & Adjusted difference ${ }^{a}$ \\
\hline Age, years & $73 \pm 8$ & $77 \pm 8$ & $4(1 ; 6)$ \\
\hline Education, primary only $\%$ & 30 & 38 & $7(-8 ; 20)$ \\
\hline Memory performance, Z-score & $0.03 \pm 0.92$ & $-0.51 \pm 0.80$ & $-0.33(-0.60 ;-0.06)$ \\
\hline $\mathrm{BMI}, \mathrm{kg} / \mathrm{m}^{2}$ & $26.2 \pm 3.7$ & $26.8 \pm 2.9$ & $0.7(-0.5 ; 1.8)$ \\
\hline Systolic blood pressure, $\mathrm{mmHg}$ & $146 \pm 20$ & $146 \pm 21$ & $-1(-8 ; 5)$ \\
\hline Total cholesterol, $\mathrm{mmol} / \mathrm{l}$ & $5.8 \pm 1.0$ & $5.9 \pm 1.3$ & $0.2(-0.2 ; 0.5)$ \\
\hline Carotid plaques, total number & $1.6 \pm 1.6$ & $2.6 \pm 1.6$ & $0.7(0.2 ; 1.3)$ \\
\hline Intima-media thickness, $\mathrm{mm}$ & $0.86 \pm 0.14$ & $0.93 \pm 0.13$ & $0.04(-0.01 ; 0.08)$ \\
\hline Periventricular white matter lesions, grade & $2.7 \pm 2.2$ & $3.6(2.2)$ & $0.4(-0.2 ; 1.1)$ \\
\hline Subcortical white matter lesions, $\mathrm{ml}$ & $1.7 \pm 3.4$ & $2.3(3.6)$ & $0.01(-1.0 ; 1.0)$ \\
\hline
\end{tabular}

Data are given as means \pm standard deviation or percentages

a Age and sex adjusted difference (95\% CI) in variable between participants with and without diabetes mellitus

b Present for 405 participants without diabetes

cluded persons with genotype $\varepsilon 2 \varepsilon 4(n=9)$ in the analyses considering $A P O E$ genotype. To assess carotid atherosclerosis, participants underwent ultrasonography of the carotid arteries [26]. Presence of atherosclerotic plaques was determined at the common carotid artery, the carotid bifurcation, and the internal carotid artery at the left and right side and summed (range $0-6)$. The intima-media thickness was measured by longitudinal two-dimensional ultrasound of the anterior and posterior wall of both common carotid arteries. We calculated the mean of these four locations. Cerebral white matter lesions were assessed on proton density weighted axial MR images and were scored in the periventricular regions (range 0-9) and the subcortical regions (approximated volume) [19]. Brain infarcts were defined as focal hyperintensities on T2 weighted images, and, if present in the white matter, with corresponding prominent hypointensity on T1 [23].

Data analysis. We missed information on the presence of diabetes mellitus in five participants, leaving a total of 506 participants for the analyses. We used multiple linear regression modelling to quantify the relation between diabetes, insulin resistance in non-diabetic subjects, and MRI volumes. Adjustments were made for age and sex. Additional adjustments included BMI, pack-years of cigarette smoking, blood pressure and serum cholesterol as co-variates. To investigate whether vascular disease was mediating any association between diabetes, insulin resistance and MRI volumes, we adjusted for carotid atherosclerosis, white matter lesions and brain infarcts on MRI. We repeated all analyses excluding subjects with infarcts on MRI. Finally, because the effect of diabetes on the risk of dementia might differ across $A P O E$ genotypes [4], we studied possible effect modification by $A P O E$ genotype through stratified analyses (non-carrier of the $\varepsilon 4$ allele versus carrier of the $\varepsilon 4$ allele). Assumptions of the model were verified by residual diagnostics. A $p$ value of less than 0.05 was considered to be statistically significant.

\section{Results}

Selected characteristics of the study cohort according to the presence of diabetes mellitus are given in Table 1 . In total, 41 participants $(8.1 \%)$ had diabetes mellitus. Twenty-six of them were treated with antidiabetic medication at time of MRI. Their median age when they were diagnosed with diabetes mellitus was 64 years (range 43-84), suggesting that they all had Type 2 diabetes. Of note, although all participants were clinically free from dementia, persons with diabetes mellitus performed worse on memory tests (Table 1). Persons with diabetes mellitus had more atherosclerotic plaques in the carotid arteries (Table 1). They also had more cerebral white matter lesions on MRI, but this was not statistically significant after accounting for age and sex differences. Brain infarcts were 1.7 times (95\% CI 0.8 to 3.3) more frequent in subjects with diabetes mellitus compared to those without diabetes mellitus, after adjusting for age and sex.

Subjects with diabetes mellitus had smaller hippocampal and amygdalar volumes on MRI (Fig. 2). Diabetes mellitus had a similar effect on the left and the right-sided brain volumes separately. Additional adjustments for BMI, pack-years of cigarette smoking, blood pressure and cholesterol, did not change the results. Although subjects with diabetes mellitus had more vascular disease, accounting for markers of vascular disease did not change the association between diabetes and hippocampal or amygdalar volumes (Table 2). Exclusion of participants with infarcts ( $n=142$ ) did not change the results either. There was no difference in association between diabetes and 

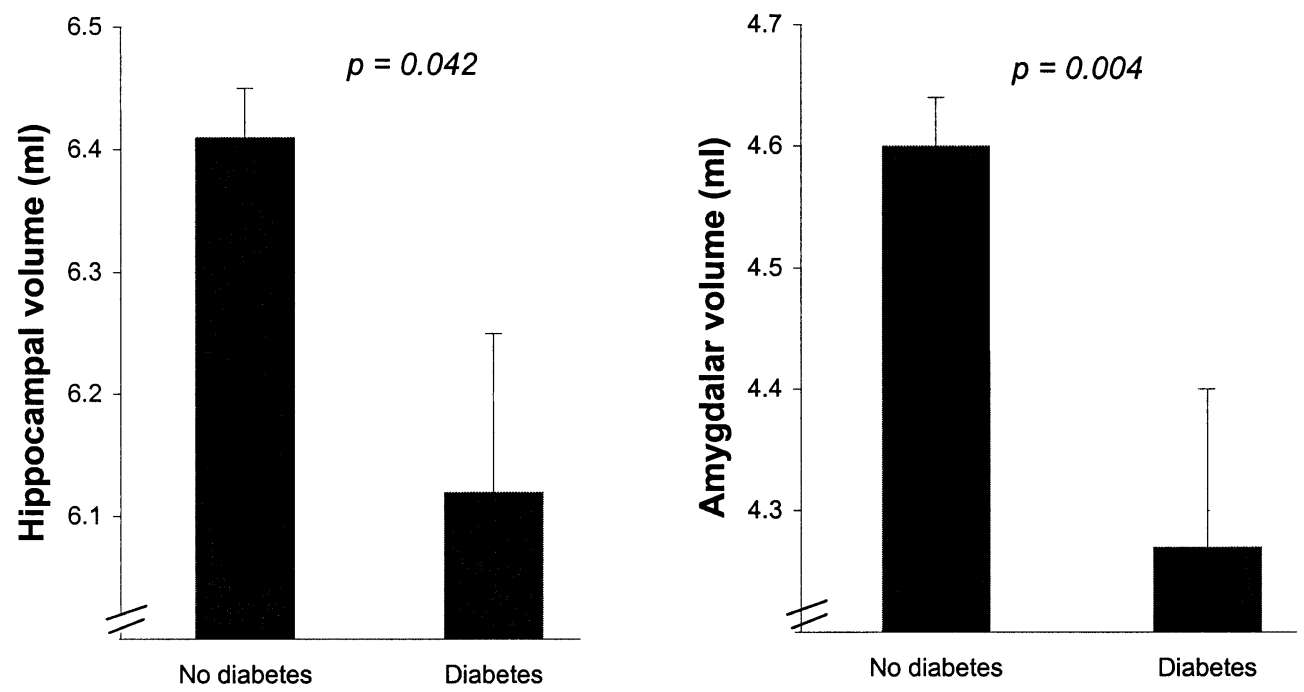

Fig. 2. Hippocampal volumes and amygdalar volumes (+standard error) on brain MRI in participants with diabetes $(n=41)$ and without diabetes $(n=465)$. Volumes are adjusted for age and sex and normalised to average head size

Table 2. Hippocampal and amygdalar volume on MRI in participants with and without diabetes mellitus accounting for markers of vascular disease

Difference adjusted for

Volume difference between participants with and without diabetes mellitus, ml (95\% CI)

\begin{tabular}{lllll} 
& Hippocampus $^{\mathrm{a}}$ & $p$ & Amygdala $^{\mathrm{a}}$ & $p$ \\
\hline Age and sex & $-0.28(-0.55$ to -0.01$)$ & 0.042 & $-0.33(-0.55$ to -0.11$)$ & 0.004 \\
Age, sex, and carotid atherosclerosis & $-0.27(-0.55$ to 0.00$)$ & 0.053 & $-0.32(-0.54$ to -0.10$)$ & 0.005 \\
Age, sex, white matter lesions and infarcts on MRI & $-0.27(-0.54$ to 0.00$)$ & 0.053 & $-0.33(-0.56$ to -0.11$)$ & 0.003 \\
\hline
\end{tabular}

Data are given as adjusted differences (95\% CI and $p$ value) in MRI volumes (ml) between participants without diabetes mellitus $(n=465)$ and participants with diabetes mellitus $(n=41)$

a Volumes are normalised to average head size

MRI volumes according to $A P O E$ strata. In non-carriers of the $\varepsilon 4$ allele, the age and sex adjusted difference in hippocampal volume between persons with and without diabetes mellitus was -0.30 (95\% CI -0.66 to $0.06)$. In carriers of the $\varepsilon 4$ allele the difference in hippocampal volume was -0.31 (95\% CI -0.84 to 0.21$)$. In non-carriers of the $\varepsilon 4$ allele, the age and sex adjusted difference in amygdalar volume between persons with and without diabetes mellitus was -0.28 (95\% CI -0.58 to 0.01$)$. In carriers of the $\varepsilon 4$ allele the difference in amygdalar volume was $-0.41(95 \% \mathrm{CI}-0.86$ to 0.04$)$.

In non-diabetic participants $(n=465)$, post-load insulin concentrations and insulin resistance were present for 405 participants. Persons with higher post-load insulin concentrations or insulin resistance had smaller amygdalar volumes, but not smaller hippocampal volumes on MRI (Table 3). Additional adjusting for BMI, pack-years of cigarette smoking, blood pressure, cholesterol, carotid atherosclerosis, white matter lesions and infarcts did not change the associations, nor did excluding participants with infarcts. The association between insulin resistance and amygdalar vol- umes on MRI was similar in APOE strata, although statistically significant only in non-carriers of the $\varepsilon 4$ allele [non-carriers: adjusted difference in amygdalar volume per standard deviation increase in insulin resistance $-0.11(95 \% \mathrm{CI}-0.19$ to -0.04$)$; carriers: -0.02 (95\% CI -0.18 to 0.15$)]$.

\section{Discussion}

We observed that people with Type 2 diabetes had more hippocampal and amygdalar atrophy on MRI than people without diabetes. Moreover, in persons without diabetes mellitus, insulin resistance was associated to amygdalar atrophy on MRI. The presence of atherosclerosis or cerebrovascular disease did not explain the associations.

The strengths of our study are its population-based design and the large sample with volumetric MRI. The prevalence of diabetes mellitus in our study was comparable to another Dutch population study [27], leading to a moderate number of people with diabetes mellitus studied in the sample. However, the associa- 
Table 3. Insulin resistance in participants without diabetes $(n=405)$ in relation to hippocampal and amygdalar volumes on MRI

Volume difference per SD increase in insulin concentrations and insulin resistance, $\mathrm{ml}(95 \% \mathrm{CI})$

Hippocampus $^{\mathrm{a}} \quad p \quad$ Amygdala $^{\mathrm{a}} \quad p$

Post-load insulin (per SD)

Adjusted for

Age and sex

Age, sex and carotid atherosclerosis

Age, sex, white matter lesions and infarcts on MRI

Insulin resistance (per SD)

Adjusted for

Age and sex

Age, sex and carotid atherosclerosis

Age, sex, white matter lesions and infarcts on MRI

$$
\begin{aligned}
& -0.02(-0.11 \text { to } 0.06) \\
& -0.03(-0.11 \text { to } 0.05) \\
& -0.02(-0.11 \text { to } 0.06)
\end{aligned}
$$

$-0.08(-0.14$ to -0.01$)$

-0.08 ( -0.14 to -0.01$)$

-0.08 ( -0.15 to -0.02$)$
0.020

0.018

0.013

Data are given as adjusted differences in hippocampal or amygdalar volume on MRI (ml, 95\% CI) per standard deviation (SD) increase in post-load insulin concentration and insulin resistance

a Volumes are normalised to average head size

tions were robust and statistically significant suggesting that we had sufficient power. A limitation of our study was the indirect assessment of insulin resistance through calculating the ratio of post-load insulin concentrations with glucose concentrations. This ratio however in non-diabetic subjects correlates well with the degree of insulin resistance assessed with precise clamping techniques [28].

Several studies have found an increased risk of Alzheimer's disease in people with diabetes mellitus $[3,4,17,29,30,31]$. Other studies did not find this association or merely an association between diabetes mellitus and vascular dementia [32, 33, 34, 35]. Difficulties in diagnosing Alzheimer's disease in life and distinguishing it from vascular dementia could have resulted in different findings across studies. Alzheimer's disease is generally characterised by slow progression in clinical symptoms which is thought to reflect gradual development of the specific Alzheimer pathology over time [8]. The pathological hallmarks of Alzheimer' s disease, neurofibrillary tangles and amyloid plaques, occur in the most early stage of the disease in the hippocampus and amygdala [8] causing neuronal loss and atrophy that can be visualised on MRI [36]. At this stage, dysfunction of the hippocampus could cause memory impairment, a well known early neuropsychological sign of Alzheimer's disease [37]. In our study, we used hippocampal and amygdalar atrophy on MRI in elderly subjects who were clinically free of dementia as markers of pre-clinical Alzheimer's disease. Several studies including our own [20] show that people with hippocampal atrophy on MRI have lower verbal memory performance [38]. Moreover, besides memory impairment, people with hippocampal atrophy on MRI frequently develop other symptoms of Alzheimer's disease later in life [11,
12]. To our knowledge, no study has prospectively examined the specific role of amygdalar atrophy in Alzheimer's disease. However, patients with very mild Alzheimer's disease have equal volume losses in the hippocampus and amygdala on MRI compared to control subjects $[13,14]$ suggesting that atrophy of both hippocampal and amygdalar are early MRI markers of incipient Alzheimer's disease.

Three biological explanations support an association between diabetes mellitus and hippocampal and amygdalar atrophy on MRI. First, diabetes mellitus leads to vasculopathy and changes in lipid metabolism, which in turn could be associated to hippocampal and amygdalar atrophy. Although we found a clear association between diabetes mellitus and carotid atherosclerosis, the relation between diabetes and cerebrovascular disease, as noted by the degree of white matter lesions and brain infarcts on MRI, was not very strong. Moreover, when we accounted for vascular disease the relation between diabetes mellitus and atrophy on MRI remained the same. This is in line with a recent study in people free of cerebrovascular disease that showed impaired glucose tolerance to relate to hippocampal atrophy on MRI [39]. Thus, although diabetes mellitus is a vascular risk factor, other non-vascular pathways seem to play a role in the findings. A second biological explanation is that hyperglycaemia in diabetic patients is directly associated to hippocampal and amygdalar atrophy. A prospective study found that subjects with diabetes mellitus have increased amyloid plaques and neurofibrillary tangles in the hippocampus at autopsy [4], though another post-mortem study did not [40]. In diabetic subjects, accelerated formation of advanced glycation endproducts can cross-link amyloid proteins leading to aggregation into the amyloid plaques 
$[6,41]$. In addition, glycation of the microtubule associated protein-tau could lead to formation of neurofibrillary tangles [42]. Pointing to a third biological explanation was the finding that peripheral insulin resistance was associated to amygdalar atrophy on MRI. Insulin resistance is characterised by high plasma insulin concentrations and relatively normal glucose concentrations. Patients with Alzheimer's disease have higher plasma insulin concentrations compared with control subjects $[43,44]$ but lower cerebrospinal-fluid insulin concentrations [44]. This suggests that insulin transport from plasma to the brain is diminished in Alzheimer patients [44]. Other investigations report that dysfunction of insulin signal transduction is involved in Alzheimer's disease [7, 45]. Genetic variability in genes encoding for components of the insulin-signalling pathway is associated to Alzheimer's disease [46]. Insulin regulates metabolism of amyloid proteins, prevents tau phosphorylation [7] and promotes neuronal survival [47], all actions that in case of dysfunction of the insulin pathway can lead to Alzheimer's disease. Furthermore, the insulin-degrading enzyme is, in addition to its role in degrading insulin, important in cleaving amyloid protein in the brain [48]. Mice with hypo-function of this enzyme have increased accumulation of amyloid in the brain and increased plasma insulin concentrations, further supporting a connection between insulin and Alzheimer's disease [49]. It is postulated that patients with Alzheimer's disease try to compensate for impaired insulin signalling by increasing the amount of insulin receptors [50]. Interestingly, we found a restricted relation between insulin resistance and amygdalar atrophy on MRI. Higher plasma insulin concentrations or insulin resistance were not related to hippocampal atrophy on MRI, in agreement with others [39]. The hippocampus and amygdalar differ according to the amount of insulin receptors, the hippocampus having a higher density [51]. A speculative explanation for the absence of a relation between insulin concentrations and hippocampal volumes is that the high density of insulin receptors in combination with high plasma insulin concentrations compensates for dysfunctions in the insulin-signalling pathway. However, the differential effects of insulin on the hippocampus and amygdala have yet to be confirmed.

In summary, in this community sample we found that people with Type 2 diabetes have smaller hippocampal and amygdalar volumes on MRI, supporting the view that diabetes is a risk factor for Alzheimer's disease. Since atherosclerosis or cerebrovascular disease were not explaining the associations, it is likely that direct metabolic effects of diabetes mellitus are involved. Our finding that insulin resistance was associated to amygdalar atrophy on MRI is in line with suggestions that dysfunction of insulin signalling is involved in the pathogenesis of Alzheimer's disease.
Acknowledgements. This research was financially supported by the Netherlands Organisation for Scientific Research (NWO) and the Health Research and Development Council (ZON). We thank F. Hoebeek and Dr E. Achten for their help in collecting the data.

\section{References}

1. Goldstein LB, Adams R, Becker K et al. (2001) Primary prevention of ischemic stroke: a statement for healthcare professionals from the Stroke Council of the American Heart Association. Circulation 103:163-182

2. Hébert R, Lindsay J, Verreault R et al. (2000) Vascular dementia: incidence and risk factors in the Canadian study of health and aging. Stroke 31:1487-1493

3. Ott A, Stolk RP, van Harskamp F et al. (1999) Diabetes mellitus and the risk of dementia: the Rotterdam Study. Neurology 53:1937-1942

4. Peila R, Rodriguez BL, Launer LJ (2002) Type 2 diabetes, APOE gene, and the risk for dementia and related pathologies: the Honolulu-Asia Aging Study. Diabetes 51:12561262

5. Snowdon DA, Greiner LH, Mortimer JA et al. (1997) Brain infarction and the clinical expression of Alzheimer disease. The Nun Study. JAMA 277:813-817

6. Vitek MP, Bhattacharya K, Glendening JM et al. (1994) Advanced glycation end products contribute to amyloidosis in Alzheimer disease. Proc Natl Acad Sci USA 91:4766-4770

7. Gasparini L, Netzer WJ, Greengard P et al. (2002) Does insulin dysfunction play a role in Alzheimer's disease? Trends Pharmacol Sci 23:288-293

8. Braak H, Braak E (1991) Neuropathological stageing of Alzheimer-related changes. Acta Neuropathol 82:239-259

9. Gosche KM, Mortimer JA, Smith CD et al. (2002) Hippocampal volume as an index of Alzheimer neuropathology: findings from the Nun Study. Neurology 58:1476-1482

10. Convit A, De Leon MJ, Tarshish C et al. (1997) Specific hippocampal volume reductions in individuals at risk for Alzheimer's disease. Neurobiol Aging 18:131-138

11. Jack CR Jr, Petersen RC, Xu YC et al. (1999) Prediction of $\mathrm{AD}$ with MRI-based hippocampal volume in mild cognitive impairment. Neurology 52:1397-1403

12. Schott JM, Fox NC, Frost C et al. (2003) Assessing the onset of structural change in familial Alzheimer's disease. Ann Neurol 53:181-188

13. Callen DJ, Black SE, Gao F et al. (2001) Beyond the hippocampus: MRI volumetry confirms widespread limbic atrophy in AD. Neurology 57:1669-1674

14. Krasuski JS, Alexander GE, Horwitz B et al. (1998) Volumes of medial temporal lobe structures in patients with Alzheimer's disease and mild cognitive impairment (and in healthy controls). Biol Psychiatry 43:60-68

15. Hofman A, Grobbee DE, de Jong PTVM et al. (1991) Determinants of disease and disability in the elderly: the Rotterdam Elderly Study. Eur J Epidemiol 7:403-422

16. Breteler MMB (2000) Vascular involvement in cognitive decline and dementia. Epidemiologic evidence from the Rotterdam Study and the Rotterdam Scan Study. Ann NY Acad Sci 903:457-465

17. Ott A, Stolk RP, Hofman A et al. (1996) Association of diabetes mellitus and dementia: the Rotterdam Study. Diabetologia 39:1392-1397

18. de Leeuw FE, de Groot JC, Oudkerk M et al. (1999) A follow-up study of blood pressure and cerebral white matter lesions. Ann Neurol 46:827-833 
19. de Groot JC, de Leeuw FE, Oudkerk M et al. (2000) Cerebral white matter lesions and cognitive function: the Rotterdam Scan Study. Ann Neurol 47:145-151

20. Hackert VH, den Heijer T, Oudkerk M et al. (2002) Hippocampal head size associated with verbal memory performance in nondemented elderly. Neuroimage 17:1365-1372

21. Maunoury C, Michot JL, Caillet H et al. (1996) Specificity of temporal amygdala atrophy in Alzheimer's disease: quantitative assessment with magnetic resonance imaging. Dementia 7:10-14

22. UNESCO: International Standard Classification of Education (ISCED). Document 19C/3. Paris, 1976

23. Vermeer SE, den Heijer T, Koudstaal PJ et al. (2003) Incidence and risk factors of silent brain infarcts in the population-based Rotterdam Scan Study. Stroke 34:392-396

24. Slooter AJC, Cruts M, Kalmijn S et al. (1998) Risk estimates of dementia by apolipoprotein E genotypes from a population-based incidence study: the Rotterdam Study. Arch Neurol 55:964-968

25. Farrer LA, Cupples LA, Haines JL et al. (1997) Effects of age, sex, and ethnicity on the association between apolipoprotein E genotype and Alzheimer disease. A meta-analysis. APOE and Alzheimer Disease Meta Analysis Consortium. JAMA 278:1349-1356

26. Bots ML, Hoes AW, Koudstaal PJ et al. (1997) Common carotid intima-media thickness and risk of stroke and myocardial infarction: the Rotterdam Study. Circulation 96:1432-1437

27. Mooy JM, Grootenhuis PA, de Vries H et al. (1995) Prevalence and determinants of glucose intolerance in a Dutch caucasian population. The Hoorn Study. Diabetes Care 18:1270-1273

28. Laakso M (1993) How good a marker is insulin level for insulin resistance? Am J Epidemiol 137:959-965

29. Leibson CL, Rocca WA, Hanson VA et al. (1997) The risk of dementia among persons with diabetes mellitus: a population-based cohort study. Ann NY Acad Sci 826:422-427

30. Brayne C, Gill C, Huppert FA et al. (1998) Vascular risks and incident dementia: results from a cohort study of the very old. Dement Geriatr Cogn Disord 9:175-180

31. Yoshitake T, Kiyohara Y, Kato I et al. (1995) Incidence and risk factors of vascular dementia and Alzheimer's disease in a defined elderly Japanese population: the Hisayama Study. Neurology 45:1161-1168

32. Luchsinger JA, Tang MX, Stern Y et al. (2001) Diabetes mellitus and risk of Alzheimer's disease and dementia with stroke in a multiethnic cohort. Am J Epidemiol 154:635-641

33. MacKnight C, Rockwood K, Awalt E et al. (2002) Diabetes mellitus and the risk of dementia, Alzheimer's disease and vascular cognitive impairment in the Canadian Study of Health and Aging. Dement Geriatr Cogn Disord 14:7783

34. Curb JD, Rodriguez BL, Abbott RD et al. (1999) Longitudinal association of vascular and Alzheimer's dementias, diabetes, and glucose tolerance. Neurology 52:971-975

35. Tariot PN, Ogden MA, Cox C et al. (1999) Diabetes and dementia in long-term care. J Am Geriatr Soc 47:423429
36. Bobinski M, de Leon MJ, Wegiel J et al. (2000) The histological validation of post mortem magnetic resonance imaging-determined hippocampal volume in Alzheimer's disease. Neuroscience 95:721-725

37. Petersen RC, Smith GE, Ivnik RJ et al. (1994) Memory function in very early Alzheimer's disease. Neurology 44: $867-872$

38. Petersen RC, Jack CR Jr, Xu YC et al. (2000) Memory and MRI-based hippocampal volumes in aging and AD. Neurology 54:581-587

39. Convit A, Wolf OT, Tarshish C et al. (2003) Reduced glucose tolerance is associated with poor memory performance and hippocampal atrophy among normal elderly. Proc Natl Acad Sci USA 100:2019-2022

40. Heitner J, Dickson D (1997) Diabetics do not have increased Alzheimer-type pathology compared with agematched control subjects. A retrospective postmortem immunocytochemical and histofluorescent study. Neurology 49:1306-1311

41. Münch G, Schinzel R, Loske C et al. (1998) Alzheimer's disease-synergistic effects of glucose deficit, oxidative stress and advanced glycation endproducts. J Neural Transm 105:439-461

42. Ledesma MD, Bonay P, Colaco C et al. (1994) Analysis of microtubule-associated protein tau glycation in paired helical filaments. J Biol Chem 269:21614-21619

43. Kuusisto J, Koivisto K, Mykkänen L et al. (1997) Association between features of the insulin resistance syndrome and Alzheimer's disease independently of apolipoprotein e4 phenotype: cross sectional population based study. BMJ 315:1045-1049

44. Craft S, Peskind E, Schwartz MW et al. (1998) Cerebrospinal fluid and plasma insulin levels in Alzheimer's disease: relationship to severity of dementia and apolipoprotein E genotype. Neurology 50:164-168

45. Frölich L, Blum-Degen D, Bernstein HG et al. (1998) Brain insulin and insulin receptors in aging and sporadic Alzheimer's disease. J Neural Transm 105:423-438

46. Liolitsa D, Powell J, Lovestone S (2002) Genetic variability in the insulin signalling pathway may contribute to the risk of late onset Alzheimer's disease. J Neurol Neurosurg Psychiatry 73:261-266

47. Zhao WQ, Alkon DL (2001) Role of insulin and insulin receptor in learning and memory. Mol Cell Endocrinol 177: 125-134

48. Kurochkin IV (2001) Insulin-degrading enzyme: embarking on amyloid destruction. Trends Biochem Sci 26:421-425

49. Farris W, Mansourian S, Chang Y et al. (2003) Insulin-degrading enzyme regulates the levels of insulin, amyloid $\beta$ protein, and the $\beta$-amyloid precursor protein intracellular domain in vivo. Proc Natl Acad Sci USA 100:4162-4167

50. Frölich L, Blum-Degen D, Riederer P et al. (1999) A disturbance in the neuronal insulin receptor signal transduction in sporadic Alzheimer's disease. Ann NY Acad Sci 893:290-293

51. Schulingkamp RJ, Pagano TC, Hung D et al. (2000) Insulin receptors and insulin action in the brain: review and clinical implications. Neurosci Biobehav Rev 24:855-872 